\title{
Energy-efficient conversion of amine-cured epoxy resins into functional chemicals based on swelling-induced nanopores
}

Fei Tian, Xiong-lei Wang, Yang Yang, Wenli An, Xu Zhao, Shimei Xu and Yu-Zhong Wang

Collaborative Innovation Center for Eco-Friendly and Fire-Safety Polymeric Materials(MoE), State Key Laboratory of Polymer Materials Engineering, National Engineering Laboratory of Eco-Friendly Polymeric Materials (Sichuan), College of Chemistry, Sichuan University, 29 Wangjiang Road, Chengdu 610064, China.

Number of Pages: 4

Number of Figures:8

Number of Tables: 1

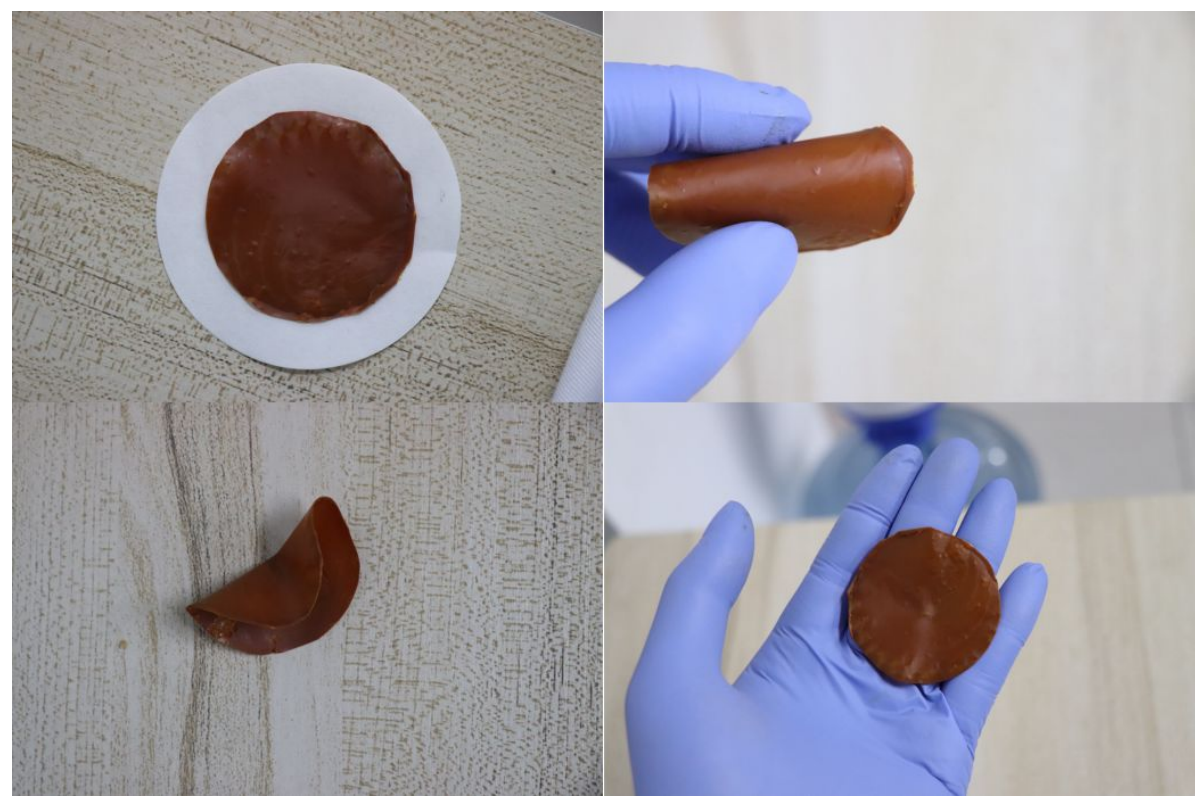

Figure S1. The DEP can be used as a self-standing membrane.

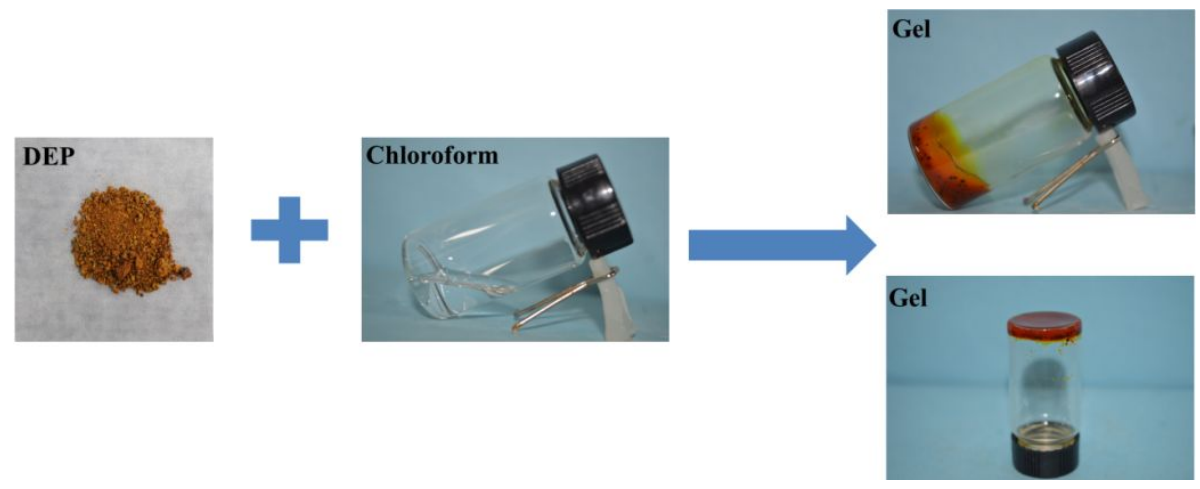

Figure S2. The DEP can be used as an oil gelator for chloroform. 


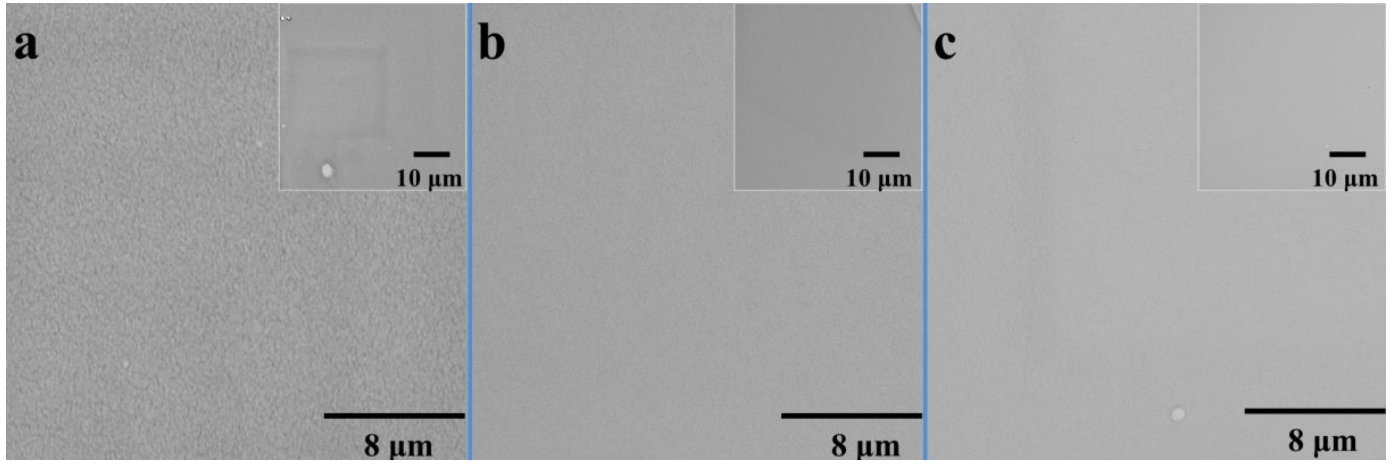

Figure S3. The SEM of EP swollen in DMA (a), DMSO (b) and DMF (c) assisted by microwave at $60^{\circ} \mathrm{C}$ for $60 \mathrm{~min}$.

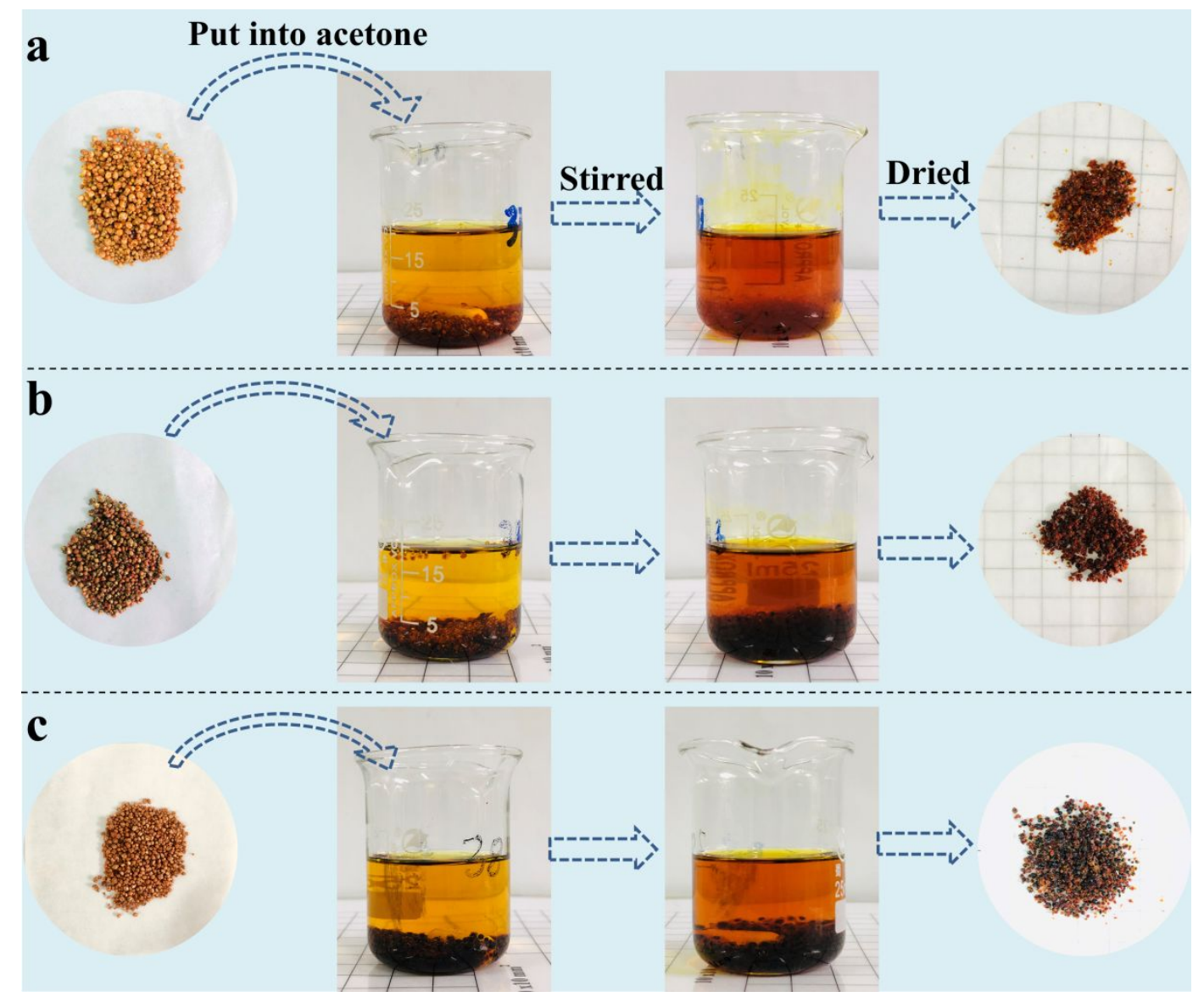

Figure S4. The optical picture of DEP, DEP dissolved in acetone and insoluble parts of DEP when the EP was swollen by DMA (a), DMSO (b) and DMF (c), respectively. 


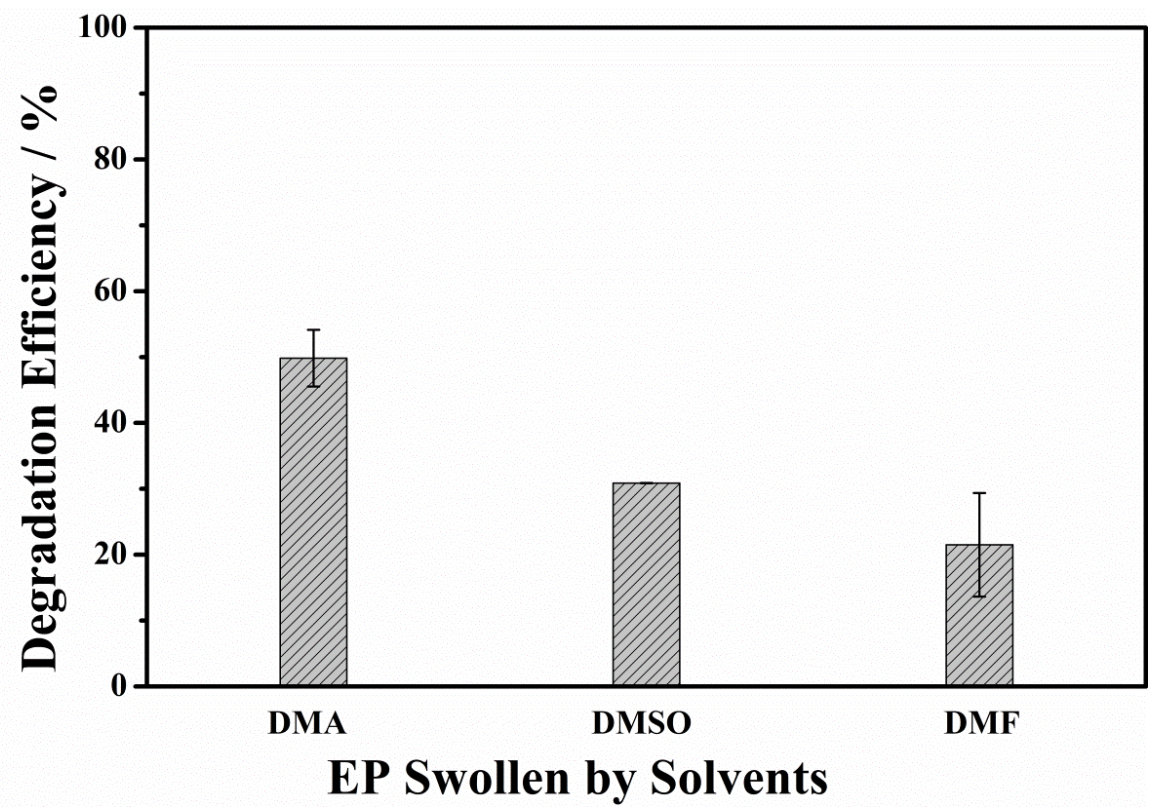

Figure S5. The degradation efficiency of EP when the EP swells by DMA, DMSO and DMF, respectively.

a
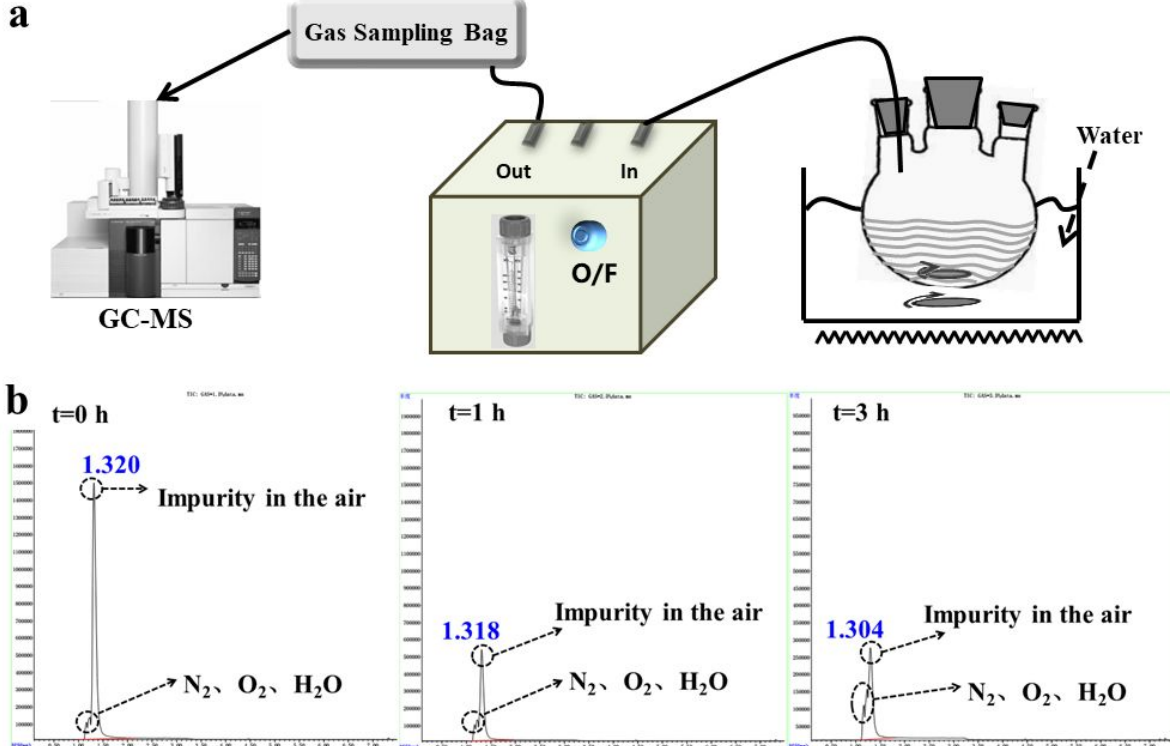

Figure S6. (a) The detection of the possible gases released in the reaction. (b) The GC-MS results of the evolution of gases from the reaction at different time. 
Table S1. The molecular weight and PDI of DEP using $\mathrm{HNO}_{3}$ incubated at $60^{\circ} \mathrm{C}$ for different reaction time and microwave irradiation time

\begin{tabular}{lcccc}
\hline & $\begin{array}{c}\text { MW Time } \\
\text { /min }\end{array}$ & $\begin{array}{c}\text { Degradation Time } \\
\text { / }\end{array}$ & Mw & PDI \\
\hline DEP-1 h & 30 & 1 & 9546 & 1.56 \\
DEP-3 h (MW 30 min) & 30 & 3 & 8800 & 1.42 \\
DEP-5 h & 30 & 5 & 8021 & 1.35 \\
DEP-10 h & 30 & 10 & 7226 & 1.25 \\
DEP-3 h (MW 10 min) & 10 & 3 & 8784 & 1.45 \\
DEP-3 h (MW 60 min) & 60 & 3 & 8282 & 1.27 \\
\hline
\end{tabular}

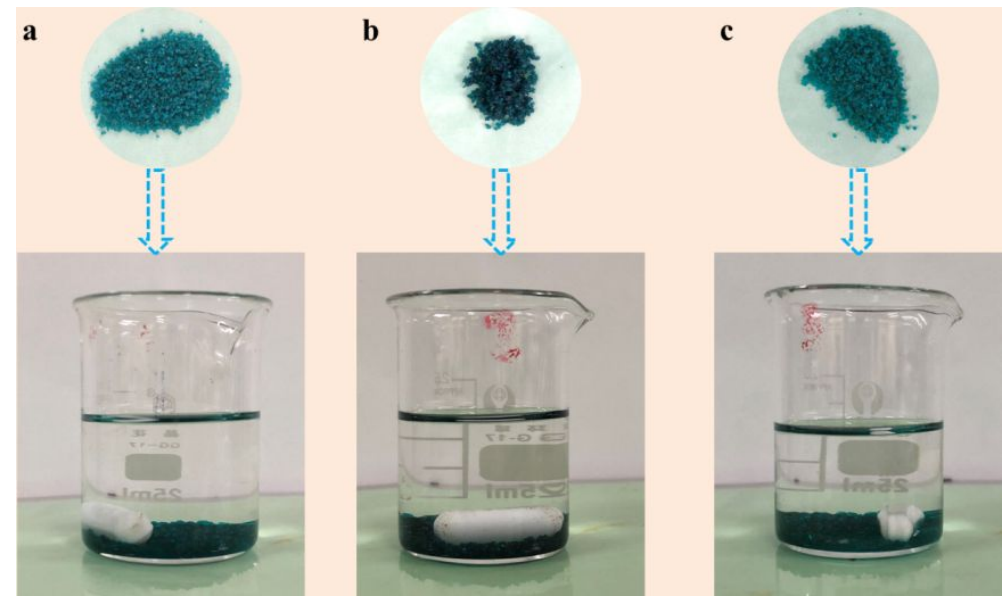

Figure S7. The degraded products of SEP (Top) by $\mathrm{HCl}$ (a), $\mathrm{H}_{3} \mathrm{PO}_{4}$ (b), and $\mathrm{H}_{2} \mathrm{SO}_{4}$ (c) at $70^{\circ} \mathrm{C}$ for $5 \mathrm{~h}$, respectively, and the degraded products put into acetone after agitating for $30 \mathrm{~min}$ (Bottom).

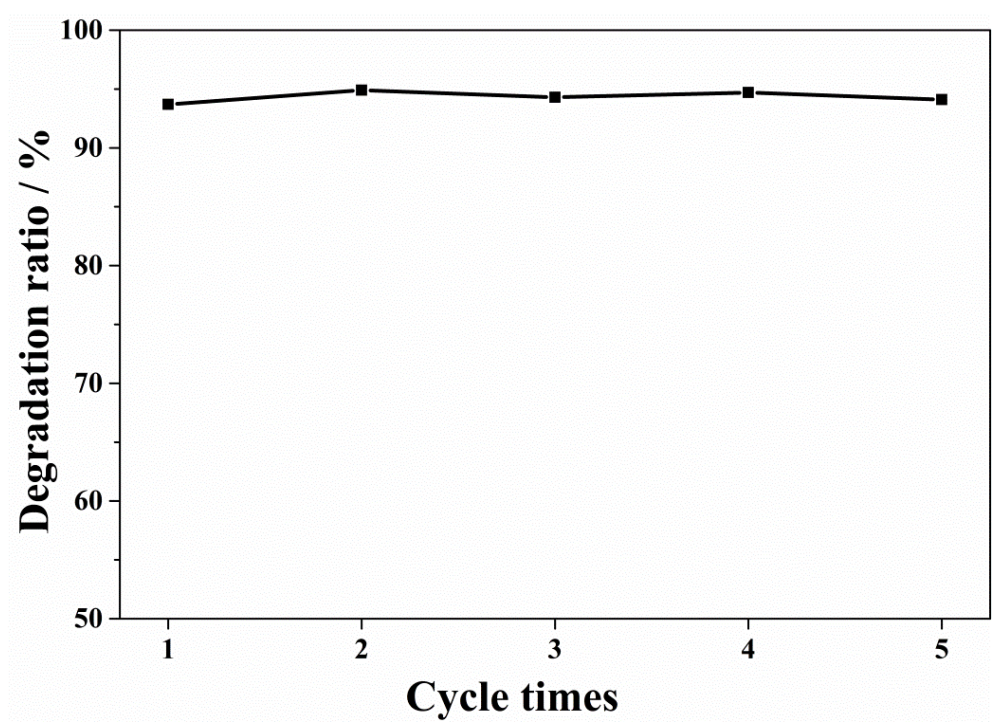

Figure S8. The degradation ratio of SEP using recycled $\mathrm{HNO}_{3}$ added with virgin $\mathrm{HNO}_{3}$ solution for 5 cycle times. 